\title{
RENDIMENTO DE CARCAÇA E CORTES DE PERUS COMERCIAIS SUBMETIDOS A DIFERENTES MANEJOS DE ALIMENTAÇÃO
}

\author{
Christine Maziero Castro ${ }^{1}$, Clovis Eliseu Gewehr ${ }^{1}$, Marcus Fernando Reginatto ${ }^{2}$ \\ 1 UDESC \\ 2 BRF \\ Correspondência: Christine Maziero Castro: chrismaziero@hotmail.com
}

\begin{abstract}
RESUMO: Avaliou-se o efeito de diferentes manejos alimentares de perus na fase de terminação sobre o rendimento de carcaça e cortes utilizando 920 machos da linhagem comercial Nicholas 700 criadas de 91 a 154 dias de idade. O experimento foi constituído de quatro tratamentos baseados na quantidade diária de ração fornecida aos perus: à vontade como testemunha (AV); controlado com fornecimento da quantidade recomendada pelo manual da linhagem (Cont); controlado em $95 \%$ da quantidade recomendada diariamente pelo manual (Cont 95) e controlado em $90 \%$ da quantidade recomendada pelo manual (Cont 90). Aos 140 e 154 dias de idade, duas aves por repetição foram abatidas e avaliados os rendimentos e pesos de carcaça e das partes. As médias dos manejos foram submetidas à análise de variância e as diferenças ao teste Tukey (5\%) e o rendimento de carcaça entre as idades ao teste $\mathrm{T}(5 \%)$. Rendimento de carcaça, perna e gordura não apresentaram diferença $(P>0,05)$ aos 140 e 154 dias. No rendimento de peito, aves submetidas ao manejo AV e Cont aos 140 dias apresentaram resultados semelhantes $(P>0,05)$ e melhores $(P<0,05)$ em relação aos demais manejos, enquanto aos 154 dias os manejos AV, Cont e Cont 95 foram semelhantes $(P>0,05)$ e apenas o Cont90 diferiu $(P<0,05)$ do AV. Peso das carcaças e partes nos manejos AV e Cont foram semelhantes $(P>0,05)$ e superiores $(P<0,05)$ aos demais, com exceção para peso de gordura que não apresentou diferença $(P>0,05)$ entre os manejos. $O$ rendimento de peito foi maior $(\mathrm{P}<0,05)$ aos 154 dias. Conclui-se que manejo de alimentação controlado com fornecimento diário da quantidade de ração recomendada pelo manual da linhagem na fase de terminação de perus de corte machos não afetam o rendimento de carcaça e das partes, no entanto, o rendimento de peito melhora aos 154 dias.
\end{abstract}

Palavras-chave: Nicholas 700; ração; rendimento de peito; fornecimento controlado de ração

\section{CARCASS AND CUTS YIELDS OF COMMERCIAL TURKEYS SUBMITTED DIFFERENT FEEDING MANAGEMENT}

\begin{abstract}
The effect of different feeding strategies was evaluated in commercial turkeys in the finishing phase over the carcass and cuts yields, utilizing 920 turkey males of Nicholas 700 breed, raised in same conditions from 91 to 154 days old. The experiment was composed of four treatments, based on daily feed consumption per bird, described as following: one ad libitum (AV) and three controlled which had different feed quantities in between each one, with the range of $100 \%$ (Cont), 95\% (Cont95) and 90\% (Cont90) of the recommendation of the breed guide. At the end of the period, 2 birds per box at 140 and 154 days were identified, weighed, slaughtered and submitted to carcass evaluation. The averages of the treatments were submitted to variance analysis, the differences to Tukey test $(5 \%)$ and the carcass yield between the ages to T test (5\%). Carcass, leg and fat yield did not differ $(P>0,05)$ on 140 and 154 days old. On breast yield, birds on treatment $A V$ and Cont on 140 days showed similar results $(P>0,05)$ and better $(P<0,05)$ compared to other treatments, while at 154 days treatments AV, Cont and Cont95 were similar $(P>0,05)$ and only Cont90 were different $(P<0,05)$ from AV. Carcass and cuts weights on treatments $A V$ and Cont were similar $(P>0,05)$ and higher than the others, except for the fat, that did not show difference between the treatments. Breast yield were higher $(\mathrm{P}<0,05)$ on 154 days. Thus, it can be concluded that controlled feeding management with daily supply of feed amount recommended by the turkey breed on the finishing phase does not affect the carcass yield and other carcass characteristics of commercial turkey males, however, breast yield improves at 154 days.
\end{abstract}

Key Words: Nicholas 700, feed, breast yield, controlled feed supply 


\section{INTRODUÇÃO}

O Brasil é o $3^{\circ}$ maior produtor e exportador mundial de carne de perus. Figuram nas primeiras posições Estados Unidos da América e a União Europeia com a soma de seus 27 membros, tanto em produção quanto em exportação. No ano de 2011 foram produzidas no Brasil 305 mil toneladas, sendo $84 \%$ destinadas aos produtos "in natura" e $16 \%$ para produtos industrializados. Deste total, 141 mil toneladas foram exportadas. Embora neste ano tenha ocorrido queda de $10,7 \%$ nas exportações, a receita cambial ainda apresentou incremento de $4,7 \%$, chegando a US\$ 444,6 milhões (UBABEF, 2012).

A produção intensiva e comercial de perus ocorre através de vários segmentos e é mais complexa quando comparada à de frangos de corte. Depender de inseminação artificial nos sistemas de produção de ovos férteis nos avozeiros e matrizeiros é um exemplo da complexidade relacionada a esta cadeia produtiva. Otimizar esta atividade no sentido de propiciar melhorias nos índices zootécnicos e de rendimento ao abate pode resultar em maiores receitas e ganhos para toda a cadeia produtiva.

Estudos que utilizam restrição quantitativa têm sido realizados com o intuito de verificar qual o melhor período para o fornecimento do alimento ou a divisão da dieta em mais de uma refeição diária (SAVORY \& MAROS, 1993; SAVORY \& KOSTAL, 1996; BACKHOUSE \& GOUS, 2006). Normalmente, uma porcentagem do consumo de ração à vontade é utilizada e deve-se pesar e fornecer a ração frequentemente, demandando mão-deobra ou sistemas automatizados para tal. Outra forma é permitir o acesso dos animais aos comedouros por um período de tempo restrito ao dia. Além disso, restrição quantitativa pode ocorrer de maneira indireta, por efeito de calor, manejo de iluminação ou restrição de água. A restrição alimentar quantitativa claramente afeta a maioria das características zootécnicas e de carcaça, e o degrau de alteração destes parâmetros depende do nível de restrição utilizada (URDANETARINCÓN \& LESSON, 2002). Este método de restrição exige espaço de alimentação adequado e fornecimento do alimento de maneira rápida para evitar desuniformidade dos animais (BUTZEN, 2012).

Melhorias na eficiência alimentar podem ser observadas com o uso de manejos de alimentação com programas de restrição alimentar (ZUBAIR \& LEESON,1994). Em frangos de corte, a restrição alimentar já foi amplamente estudada e os resultados são consistentes na produção industrial. No entanto, sabe-se que muitos são os fatores que influenciam o crescimento destas aves, incluindo a severidade, idade e duração de uma restrição alimentar (YU \& ROBINSON, 1992). Se os ganhos são evidentes em frangos de corte, pouco se sabe sobre o efeito da restrição alimentar em perus, mas especula-se que pela diferença de tamanho de ciclo de produção, algum ganho com a restrição alimentar poderá ocorrer. O que se busca como resposta é qual o impacto real no rendimento da carcaça quando de uma restrição tardia e continuada, uma vez que, de forma empírica, esta é uma prática utilizada por alguns produtores com resultados interessantes. Aliado a isto e de maneira indireta, as condições de ambiência inadequadas das instalações ou ainda o fato de em grande parte do dia os animais não consumirem ração por buscarem homeostase térmica e a digestão produzir muito calor (HAVENSTEIN et al., 2007; MAMPUTU 
et al. 1992), podem também conduzir a uma restrição alimentar.

Com base no que foi exposto, o objetivo deste trabalho foi avaliar 0 efeito de diferentes manejos alimentares em perus machos de linhagem comercial sobre 0 rendimento de carcaça e partes ao abate.

\section{MATERIAL E MÉTODOS}

O projeto constou na avaliação do rendimento de carcaça e composição corporal ao abate aos 140 e 154 dias, após criação em aviário experimental em uma Agroindústria localizada na região oeste de Santa Catarina, Brasil. Foram utilizados 920 perus machos, da linhagem Nicholas 700, alojados em 40 boxes de $8 \mathrm{~m}^{2} /$ cada, que constituíram uma unidade experimental composta por 23 aves por box. O galpão era divido por um corredor central de $5 \mathrm{~m}$, sendo 20 boxes de cada lado. As aves foram alojadas com 01 (um) dia de idade na primeira quinzena de julho de 2012, criadas em condições idênticas de acordo com o padrão da linhagem até os 91 dias de idade, quando teve início o período de avaliação, o qual teve 9 semanas de duração e encerrou-se na primeira quinzena de setembro de 2012 .

As aves foram submetidas ao fotoperíodo natural da época que foi crescente, passando de aproximadas $10 \mathrm{~h}$ para $12 \mathrm{~h}$ de luz/dia. As rações foram formuladas de acordo com as exigências nutricionais recomendadas pelo manual da linhagem (Tabela 1). $O$ experimento foi constituído de quatro manejos de alimentação (tratamentos), baseados no consumo de ração por ave, conforme estabelecido pelo manual da linhagem de acordo com a idade, sendo: à vontade (AV), onde a alimentação ficou disponível permanentemente às aves $e$ foi distribuída nos comedouros a cada dois dias; controlado 1 (Cont) onde a alimentação foi diária, com fornecimento de $100 \%$ da quantidade de ração recomendada pelo manual da linhagem de acordo com a idade, dividida e distribuída em 2 tratos diários (8 e 15h); controlado 2 (Cont95) com fornecimento diário de ração, entretanto a quantidade foi restrita em $95 \%$ do recomendado pelo manual da linhagem de acordo com a idade e controlado 3 (Cont90) com fornecimento diário de ração restrito em $90 \%$ do recomendado pelo manual da linhagem de acordo com a idade.

Tabela 1 - Composição nutricional e calculada das rações utilizadas durante o
periodo experimental para os diferentes manejos alimentares de perus
na fase de terminação.

A ração era adicionada em um comedouro tubular sendo um para cada parcela e a água foi fornecida à vontade em um bebedouro pendular em cada box. Tanto comedouros quanto bebedouros eram apropriados para a criação de perus. O manejo das aves durante o período experimental seguiu àquele recomendado pelo manual da linhagem, com exceção do manejo alimentar, o qual seguiu a descrição dos tratamentos anteriormente citados. Toda a ração fornecida foi pesada e anotada na ficha de controle de cada box, assim como todas as aves mortas foram pesadas e o apontamento da mortalidade também registrado na ficha do respectivo box. A forma física das rações seguiu o padrão utilizado pela empresa para as aves de seu plantel 
normal no período, sendo pélete de $4 \mathrm{~mm}$ com PDI de $80 \%$. O milho utilizado na produção destas rações apresentou diâmetro geométrico médio (DGM) de 750 micrômetros.

Ao final do período experimental as aves foram sacrificadas em abatedouro da mesma Agroindústria, onde metade das aves foi abatida aos 140 dias e outra metade aos 154 dias. Após seguirem-se todas as etapas normais de abate requeridas pela legislação, duas aves de cada parcela em cada idade foram selecionadas aleatoriamente para procedimento das avaliações de rendimento e peso de carcaça, peito, perna e gordura.

Utilizou-se um delineamento inteiramente casualizado, utilizando a média das duas aves de cada parcela abatidas nas diferentes idades e os resultados foram submetidos à análise de variância e quando observada diferença entre as médias dos tratamentos, estas foram comparadas pelo teste de Tukey (5\%) pelo pacote estatístico MINITAB 16 (2010). Para verificar o efeito dos tratamentos sobre o rendimento de carcaça nas diferentes idades (140 e 154 dias) foi utilizado o teste T de Student (5\%).

\section{RESULTADOS E DISCUSSÃO}

Os manejos alimentares para os perus em terminação influenciaram $(P<0,05)$ apenas $o$ rendimento de peito ao abate, sem interferência sobre rendimento de carcaça, perna e gordura, tanto aos 140 dias (Tabela 2) quanto aos 154 dias de idade (Tabela $3)$.

\begin{tabular}{|c|c|c|c|c|c|c|}
\hline & \multicolumn{4}{|c|}{ Man ejos Alimentares } & \multirow[b]{2}{*}{$E P M^{2}$} & \multirow[b]{2}{*}{ Prob $^{3}$} \\
\hline & AV & Cont & Cont95 & Cont90 & & \\
\hline Carcaça & 78,91 & 78,46 & 78,69 & 78,91 & 0,10 & NS \\
\hline Peito & $23,96^{\mathrm{a}}$ & $23,37^{\mathrm{ab}}$ & $22,97^{\circ}$ & $23,07^{\circ}$ & 0,12 & * \\
\hline Perna & 9,27 & 9,26 & 9,30 & 9,45 & 0,04 & NS \\
\hline Gordura & 0,36 & 0,41 & 0,44 & 0,38 & 0,01 & NS \\
\hline
\end{tabular}

Os resultados obtidos confirmam com os observados por Taha e Farran (2009) que relatam que as estratégias de alimentação, assim como linhagem e sexo podem influenciar a qualidade e a composição de carcaça. Ainda, de acordo com Moran et al. (1984), a proporção de peito em carcaças pós resfriamento aumenta com a idade em detrimento das demais partes. Sendo assim, a idade parece ser 0 fator dominante a afetar a distribuição de tecido muscular de peito.

Brake et al. (1995) relata que o rendimento dos componentes de carcaça é uma função de idade e sexo e que algumas destas relações com a idade não são lineares. Assim, passa a ser de fundamental importância 0 entendimento das curvas de crescimento das diferentes linhagens comerciais para o encontro da melhor relação técnica e econômica de abate, uma vez que o valor relativo dos componentes das carcaças é determinante para a lucratividade da cadeia.

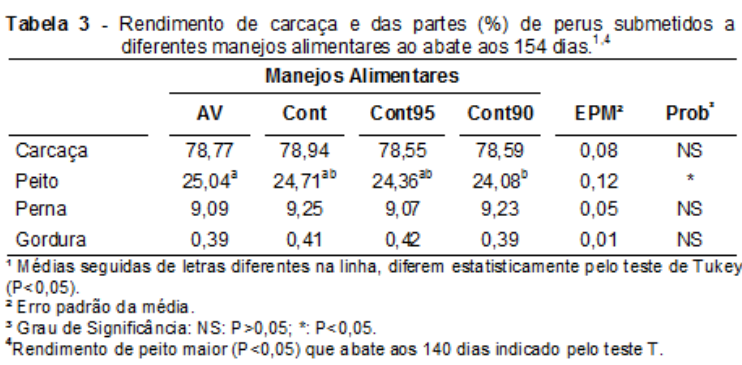

Em termos de rendimento de carcaça é importante prover uma dieta balanceada para que as aves não tenham limitação em crescimento e maximizem os resultados, especialmente em peito, que representa cerca de 26 a $28 \%$ do peso das aves e de 60 a $70 \%$ da receita econômica (HAMMOND, s/d). Assim, o rendimento de peito é o fator mais importante da carcaça de perus, sendo desta porção a matéria prima para confecção dos principais produtos comercias. Quanto maior o rendimento de peito do peru, 
maior será o seu valor comercial. Conforme o resultado obtido pelo teste $\mathrm{T}$ entre 140 e 154 dias é mais interessante abater o animal aos 154 dias. Também, observa-se que apenas - manejo alimentar controlado com fornecimento de $90 \%$ da quantidade recomendada afeta negativamente 0 rendimento de peito aos 154 dias. Já no abate aos 140 dias, os manejos controlados 90 e $95 \%$ pioraram o rendimento de peito. Esta discordância de resultado no manejo $90 \%$ entre 140 e 154 dias pode ser um indicativo de que o manejo alimentar possa interferir no rendimento da carcaça dependendo da idade que o peru é abatido.

Analisando-se 0 peso das carcaças e dos componentes, observase que os manejos alimentares para os perus em terminação influenciaram $(\mathrm{P}<0,05)$ todas as variáveis analisadas (peso de carcaça, de peito e de perna), sendo que aves do método de manejo alimentar Cont90 tiveram pior resultado e diferente estatisticamente $(P<0,05)$ dos demais (Tabelas 4 e 5) tanto aos 140 quanto aos 154 dias. $A$ única variável que não foi influenciada em nenhum método foi o peso da gordura $(P>0,05)$.

Tabela 4 - Peso de carcaça e das partes $(\mathrm{kg})$ de perus submetidos a diferentes $\frac{\text { manejos alimentares ao abate aos } 140 \text { dias'. }^{1}}{\text { Manejos Alimentares }}$

\begin{tabular}{|c|c|c|c|c|c|c|}
\hline & AV & Cont & Cont95 & Cont90 & $E P M^{2}$ & Prob $^{2}$ \\
\hline Carcaça & $16,31^{2}$ & $16,02^{2}$ & $15,70^{\mathrm{ab}}$ & $15,13^{\circ}$ & 0,090 & * \\
\hline Peito & $4,95^{\circ}$ & $4,78^{\mathrm{ab}}$ & $4,59^{\circ 0}$ & $4,42^{\circ}$ & 0,040 & * \\
\hline Perna & $1,92^{2}$ & $1,89^{\mathrm{ab}}$ & $1,86^{\mathrm{ab}}$ & $1,81^{\circ}$ & 0,014 & * \\
\hline Gordura & 0,08 & 0,09 & 0,09 & 0,07 & 0,002 & NS \\
\hline
\end{tabular}

De acordo com Brake et al (1995), o peso total da carcaça não reflete o peso dos componentes nas diferentes idades, mas o crescimento das aves segue uma curva sigmoide (ANTHONY et al., 1991) e as taxas de crescimento podem ser comparáveis entre frangos e perus, embora nestes o alcance do ponto de inflexão aconteça mais tarde e a resposta em peso possa ser influenciada pelo momento de inflexão da curva de crescimento.

Os autores não encontraram na literatura trabalhos similares para qualificar a discussão. Contudo, em função do crescimento do tecido muscular ser mais tardio e de haver demanda maior de nutrientes na fase inicial para um correto desenvolvimento de ossos e nervos, alimentar as aves com os adequados requerimentos em todas as fases de sua vida é a melhor estratégia para atingir melhores indicadores zootécnicos e de rendimento de carcaça (HAMMOND, $\mathrm{s} / \mathrm{d}$ ). A idade de abate dos perus afetará a composição final de carcaça em termos de gordura, ossos e músculos.

Assim, abater aves jovens poderá aumentar a quantidade de ossos relativo à gordura e músculo, enquanto abater aves mais velhas tende a aumentar a relação de músculo e gordura. Desta maneira, reduzir a habilidade das aves de expressar seu potencial genético na fase final de crescimento, seja via restrição de alimento ou qualquer outra alternativa, terá um efeito negativo em rendimento de carcaça (HAMMOND, $\mathrm{s} / \mathrm{d})$.

\section{CONCLUSÃO}

Manejo de alimentação controlado com fornecimento diário da quantidade de ração recomendada pelo manual da linhagem na fase de terminação de perus de corte machos não afeta o rendimento de carcaça e das partes. Manejos com redução de 5 e $10 \%$ da quantidade diária recomendada piora o rendimento de peito de perus abatidos aos 140 dias de idade. Manejo com redução de $10 \%$ da quantidade diária piora o rendimento de peito no abate aos 154 dias. O rendimento de peito de perus melhora ao abate aos 154 dias em relação a 140 dias. 

a diferentes manejos de alimentação

Novos estudos devem ser conduzidos para confirmar os resultados obtidos, bem como entender as questões relacionadas ao manejo alimentar tardio em perus comerciais machos.

\section{NOTAS INFORMATIVAS}

Este estudo foi submetido ao comitê de ética em experimentação animal da instituição de origem, sendo aprovado sob o protocolo de número 1.47 .13

\section{REFERÊNCIAS}

ANTHONY, N. B.; EMMERSON, D.A.; NESTOR, K.E., et al. Comparison of growth curves of weight selected populations of turkeys, quail, and chickens. Poultry Science, v.70, p.13-19. 1991.

BRAKE, J.; HAVENSTEIN, G. B.; FERKET, P. R., et al. Relationship of sex, strain, and body weights to carcass yield and offal production in turkeys.Poultry Science, v.74, p.161-168. 1995.

BACKHOUSE, D.; GOUS, R. M. Responses of adult broiler breeders to feeding time. World's Poultry Sci. Journ., v.62, n.2, p.269-281. 2006.

BUTZEN, F. M. Programas de restrição alimentar precoce e seu efeito sobre os índices zootécnicos e qualidade de carne de frangos de corte. 2012. 226 f. Dissertação (Mestrado em Zootecnia) - Universidade Federal do Rio Grande do Sul. Porto Alegre, 2012.

HAMMOND, L. The influence of diet on breast meat yield. Aviagen Turkeys, Tecnhical Article, s/d.

HAVENSTEIN, G. B.; FERKET, P. R.; GRIMES, J. L.; et al. Comparison of the performance of 1966- versus 2003-type turkeys when fed representative 1966 and 2003 turkey diets: growth rate, livability, and feed conversion. Poultry Science, v.86, p.232-240. 2007.

MAMPUTU, M.; CUNNINGHAM, D. L.; BUHR, R. J. Performance of turkeys subjected to day and night feeding programs during heat stress.
Journal of Applied Poultry Research, v.1, p.296-299.1992.

MINITAB 16.Minitab. 2010. Minitab, State College, PA.

MORAN, E. T.; POSTE, L. M.; FERKET, P. R., et al. Response of large tom turkeys differing in growth characteristics to divergent feeding systems: performance, carcass quality, and sensory evaluation. Poultry Science, v.63, p.1778-1792. 1984.

\section{NICHOLAS TURKEYS. Management essencials for commercial Turkeys. Disponível em \\ <http://www.aviagenturkeys.com/us/document- library.aspx > Acesso em 03 mar. 2013. \\ SAVORY, C. J.; KOSTAL, L. Temporal patterning of oral stereotypies in restricted-fed fowls: 1 . Investigations with a single daily meal. International Journal of Comparative Psychology, v.9, n.3, p.117-139. 1996.}

SAVORY, C. J.; MAROS, K. Influence of degree of food restriction age and time of the day on behavior of broiler breeder chickens.

Behavioural Processes, v.29, p.179-190. 1993.

TAHA, N. T.; FARRAN, M. T. Comparative study of thigh muscles and bones conformation and some carcass traits of local vs. imported turkey strain. International Journal of Poultry Science, v.8, n.4, p.368-372. 2009.

UBABEF. União Brasileira de Avicultura. Relatório anual 2012. São Paulo: 2012. 113p.

URDANETA-RINCÓN, M.; LEESON, S. Quantitative and qualitative feed restriction on growth characteristics of male broiler chickens. Poultry Science, v.81, p.679-688. 2002.

YU, M. W.; ROBINSON, F. E. The application of short-therm feed restriction to broiler chicken production: a review. Journal of Applied Poultry Research, v.1, p.147-153.1992.

ZUBAIR, A. K.; LEESON, S. Effect of early feed restriction and realimentation on heat production and changes in sizes of digestive organs of male broilers. Poultry Science, v.73, p.529-538. 1994. 